\title{
Damage Control Surgery for Exsanguinating Abdominal Trauma Patients
}

\author{
Ahmed Kamal Gabr, MD; Hossam M. Saleh, MD.
}

Vascular Surgery Department, Ain Shams University, Cairo, Egypt.

Background/Aim: Damage control surgery (DCS) has become a well-established in the past few decades as a surgical strategy to be applied in the unstable trauma patients. Damage control surgery, sometimes known as "damage limitation surgery" or "abbreviated laparotomy, is best defined as creating a stable anatomical environment to prevent the patient from progressing to an unsalvageable metabolic state. Patients are more likely to die from metabolic failure or the lethal triad (hypothermia, metabolic acidosis and coagulopathy) than from failure to complete organ repairs. The aim of this study was to analyze the role of damage control surgery in abdominal trauma patients in terms of morbidity and mortality.

Patients and methods: A retrospective review of all patients undergoing a laparotomy and damage control surgery in a level 1 trauma center over a 3-year period was performed. This study includes 42 severely injured patients who presented in the emergency room of a tertiary referral hospital in the eastern province in Saudi Arabia. These patients were hemodynamically unstable because of life-threatening hemorrhage following either blunt or penetrating abdominal trauma. After stat shifting to the operating theatre, both resuscitation and operative intervention were done simultaneously. Variable procedures of damage control surgery like abdominal packing for hepatic and pelvic trauma, major abdominal vessel ligation and temporary shunting using silastic tubes for vascular injury were done in phase I. In phase II patients were managed in the surgical intensive care unit (SICU) for hypothermia, acidosis, and coagulopathy. Phase III for definitive treatment was done after 24-72 hours once the patients got stable.

Results: Over the duration of this 3-year study, 42 patients underwent a damage control laparotomy following trauma. There were 93 organ injuries in these 42 patients. The mechanism of injury was blunt trauma in 31 patients (74\%), stab wound in 7 patients (17\%) and gunshot wounds in 4 patients (9.5\%), 28 patients (66.7\%) had been involved in motor vehicle accidents and 3 patients (7\%) are involved in fall from height. Average time interval between presentation in emergency department and surgical intervention was 17 minutes, and average operating time was 50 minutes. Twenty patients died, giving an overall mortality rate of $47.6 \%$. The mean age of the patients who survived was 24 years, compared with 36 years in the non-survivor group. Increasing age was found to be a statistically significant factor predicting mortality, with a p-value of 0.001. The development of DIC $(p<0.001)$, the need for inotropes $(p<0.001)$ and the presence of septic shock ( $p=0.017)$ were found to be significant predictors of mortality.

Conclusion: Damage control surgery still represents an important refuge to reduce morbidity and mortality in trauma resuscitation as it gives the patient a chance to survive in an otherwise hopeless situation. The results obtained from our study are in accordance with other studies published to-date i.e. Reducing mortality and morbidity in addition to an improved outcome. The management of this complex problem requires a multidisciplinary team approach with patient counseling and communication with the family.

Key words: Damage control surgery, abbreviated laparotomy, abdominal packing, open abdomen, hypothermia, coagulopathy. 


\section{Introduction:}

Damage control surgery, "abbreviated laparotomy", "staged laparotomy", "temporary abdominal closure" are synonymous. Over the past two decades, damage control surgery (hereafter, DCS) rather than definitive repair of all injuries has become established as the appropriate surgical strategy in the severely injured patient needing operative intervention. ${ }^{1}$ The term "damage control surgery" was first described in trauma by Rotondo and Schwab, who in 1993 outlined a three-phase approach to patients with major abdominal injuries. ${ }^{2}$ Damage control is defined as the rapid initial control of hemorrhage and contamination, temporary closure, resuscitation to normal physiology in the ICU, and subsequent reexploration and definitive repair. ${ }^{2,3}$ Damage control is applied when the initial laparotomy is ended and expeditious indirect methods are applied to control massive bleeding or soil or both. 4,5 This has increased the survival rate after major trauma to over $50 \%$. These comprise the first stage, namely the decision as to when to perform DCS, and the final stage of abdominal wall closure. ${ }^{6}$ However, little appears to have been documented on factors predicting mortality in this setting. In essence, damage control surgery equates with abbreviated surgery and restoration of near physiology, in a staged approach to a life-threatening injury. ${ }^{7}$ This study reviews the experience of damage control surgery for exsanguating abdominal trauma patients in terms of care and complications.

\section{Patients and methods:}

Forty-two patients who presented in the emergency room of a Almoosa Specialized Hospital, Al-Ahsa, Saudi Arabia, who underwent DCS for abdominal injury were retrospectively reviewed using the trauma registry of our level I trauma center to review all patients from 1st September 2010, through $31^{\text {st }}$ August 2013. This included management of solid organ injuries by packing, resection of gastrointestinal tract injuries without re-anastomosis, major vessel temporary shunting using silastic tubes for arterial injuries to restore distal lower limb perfusion during the patient's stay in SICU or ligation of the venous injury, and use of temporary abdominal closure techniques. All patients admitted were resuscitated in accordance with treatment protocols outlined in the Advanced Trauma Life Support course (ATLS) ${ }^{8}$ of the American College of Surgeons. Damage control surgery was defined as an abbreviated laparotomy performed either because of poor physiological status or the extent of the injury caused by the trauma, with definitive surgery to be performed 24-72 hours later after resuscitation in the ICU.

Inclusion criteria:

- Exsanguating abdominal trauma patients.

- Haemodynamically unstable patients.

- Early blood loss of 4-5 litres.

- Arterial pH of 7.25 or less.

- A core body temperature of $34^{\circ} \mathrm{C}$.

- Evidence of disseminated intravascular coagulation (DIC).

Nevertheless, these inclusion criteria were somewhat arbitrary because they may involve borderline physiological conditions in which the patient's survival may be unlikely even with damage control. A decision to proceed should be made as soon as the extent of the visceral damage has been assessed. Nevertheless, the surgeon should not feel reluctant to use damage control at any time when needed. Early abbreviated laparotomy is done for the patient who can be only partly resuscitated to curtail life-threatening haemorrhage and minimize further major peritoneal soiling.

\section{Exclusion criteria:}

- Age 70 years and more.

- Fatal head injury patients.

- Pre-hospital cardiac arrest.

As soon as the patient met the inclusion criteria for abbreviated laparotomy as above mentioned reached the emergency room of our trauma 1 center, immediate regulations for shifting to operating room and surgical interventions were made. Simultaneous resuscitation and surgical intervention were started on the operation table. Abdominal 
packing for hepatic trauma and pelvic injury, major vessel shunting using silastic tubes or major vessel ligations were utilized whenever required in these exsanguating abdominal trauma victims. We utilized different techniques of temporary abdominal closure, e.g. Towel clips, one layer continuous suture, or Bogota's technique using sterile uribag Figure (1). After phase I of damage control is accomplished, the patient is to be shifted to the surgical ICU to be enrolled in phase II for prevention or correction of the trauma triad of death i.e. Hypothermia, coagulopathy and acidosis.

In the surgical ICU, re-warming, correction of coagulopathy, acidosis and optimization of the pulmonary functions were performed. Once the patient got stable in the phase II of damage control approach, planned return to the operating room was made for review of injuries, removal of packs, removal of temporary vascular shunts and definitive vascular repair, debridement of ischaemic necrotic tissues, assessment of viability of tissues, definitive treatment of other injuries previously left untreated and proper abdominal closure if feasible.

Medical records were maintained and later on reviewed for degree and pattern of injury, transfusion requirements for preoperative and postoperative phases, resuscitation and operative time, $\mathrm{pH}$ and bicarbonates, complications and definitive treatment and mortality.

\section{Results:}

Over the duration of this 3-year study, 42 patients underwent a damage control laparotomy following trauma. The mean age of these patients was 29 years (range $16-58$ years). There were 34 males (81\%) and 8 females (19\%). There were 93 organ injuries in these 42 patients, distributed as set out in Table (1). The mechanism of injury was blunt trauma in 31 patients $(74 \%)$, stab wound in 7 patients $(17 \%)$ and gunshot wounds in 4 patients $(9.5 \%), 28$ patients $(66.7 \%)$ had been involved in motor vehicle accidents and 3 patients (7\%) are involved in fall from height. Average time interval between presentation in emergency department and surgical intervention was 17 minutes, and average operating time was 50 minutes. Twenty patients died, giving an overall mortality rate of $47.6 \%$. The mortality rates for gunshot wounds, blunt trauma and stab wounds were $35 \%, 32.5 \%$ and $5 \%$, respectively. Seven patients $(16.7 \%)$ died in the surgical ICU within 24 hours after the initial damage control laparotomy. An emergency re-look was necessary in 13 patients (31\%) after a mean of 12 hours because of bleeding in 7 patients, abdominal compartment syndrome in 5 patients and bowel leakage in 1 patient. Of these patients, 5 died within the next 2 weeks. Twenty seven patients (64.3\%) underwent a planned re-look after a mean of 41.6 hours, and $5(18.5 \%)$ of these patients died. Three patients $(7.1 \%)$ died within 1 week during phase II DCS during their stay in SICU, 1 from cardiac failure, 1 from pulmonary embolism, and 1 from the associated head injuries. The total number of deaths during the entire period of study was 20 patients (47.6\%). The mean age of the patients who survived was 24 years, compared with 36 years in the non-survivor group. Increasing age was found to be a statistically significant factor predicting mortality, with a p-value of 0.001 . Twenty three $(54.76 \%)$ patients developed DIC, twenty patients $(47.6 \%)$ required inotropes, 15 patients $(35.7 \%)$ were diagnosed with systemic inflammatory response syndrome (SIRS), 10 patients (23.8\%) developed abdominal compartment syndrome, 7 patients $(16.7 \%)$ were treated for nosocomial pneumonia, and 3 patients (7.1\%) were treated for septic shock. The development of DIC $(p<0.001)$, the need for inotropes $(p<0.001)$ and the presence of septic shock $(p=0.017)$ were found to be significant predictors of mortality.

\section{Discussion:}

The premise of damage control laparotomy is that the metabolic derangement of ongoing bleeding supersedes the need for definitive operation. As such, the main thrust of damage control laparotomy is the rapid surgical control of bleeding. Damage control 
laparotomy has led to better outcomes than expected in these grievously injured patients. Experience with high volume of severely injured casualties expedites the process. Historically these conditions have converged during times of conflict, improving the care of combat casualties and subsequently that of civilian trauma patients. ${ }^{9}$

The conventional sequence of the management of trauma surgery was to bring the patient to the operating room after initial resuscitation and then to operate for complete repair of the injuries. Even patients with multiple complex injuries were operated more aggressively over a prolonged period of time for definitive primary repair10. Subsequently, these patients were sent to the intensive care unit where a good number of the patients succumbed due to metabolic derangement of the body. ${ }^{11,12,13}$

In the most severely injured casualties, it is well-established that when the lethal triad of hypothermia, acidosis, and coagulopathy is present, death is imminent. Current practice is to avoid reaching these conditions by using damage control surgery. However conventional resuscitation practice for damage control focuses on rapid reversal of acidosis and prevention of hypothermia, and surgical techniques focus on controlling hemorrhage and contamination. ${ }^{14}$ Direct treatment of coagulopathy has been relatively neglected, viewed as a byproduct of resuscitation, hemodilution, and hypothermia, and delayed by banking logistics. ${ }^{15}$ Damage control resuscitation addresses the entire lethal triad immediately upon admission in hospital and as a structured intervention begins immediately after rapid initial assessment in the emergency department and progresses through or into ICU.16,17

At the first phase of damage control strategy, only abbreviated laparotomy was done for lifesaving measures, and then the patient was sent to the surgical intensive care unit (SICU) for the correction of the metabolic disorders. Following satisfactory correction the patient was once again taken to the operation room for definitive repair and sent back to SICU for further convalescence, which had been done in our study.

Staged surgical procedures including staged laparotomy represent an important development in the historical spectrum of trauma resuscitation. ${ }^{17,18}$ Successful damage control operations are currently best to save life in experienced hands. Management of these complex patients requires an effort from all members of a multidisciplinary trauma team. Rontodo MF along with his team proved twice in his studies the role of damage control surgery and its logic and concluded that damage control is a safe approach for increased survival in exsanguinating patients with major vascular and multiple visceral penetrating abdominal injuries. ${ }^{14,19}$ Optimal management involves rapid homeostasis and reversal of metabolic derangements utilizing damage control principles. The traditional concept of damage control surgery favors a life over limb approach and discourages elaborate, prolonged vascular reconstruction. However limb preservation could be successful when the control approach is combined with advanced resuscitative strategies and vascular techniques. Gillespie DL and his team suggested that aggressive damage control resuscitation maneuvers in critically injured casualties successfully permitted prolonged, complex extremity revascularization with excellent limb salvage and graft patency. Recombinant VIIa, fresh frozen plasma, fresh whole blood, platelets and cryoprecipitate, while minimizing crystalloids allowed limb salvage and did not result in early graft failures. ${ }^{20} \mathrm{We}$ also have seen in our study that this approach has made us able to save even the severely injured patients of polytrauma. A comparison of our patient population with previous report on damage control from Rotondo et al. The overall survival rate $58 \%$ in Rotondo et al. While in our study was $52.4 \%$.

Overall results obtained from this study are broadly consistent with the other studies published to-date, i.e. Reducing morbidity and mortality, and improving outcome.

\section{Conclusion:}

Damage control surgery still represents 


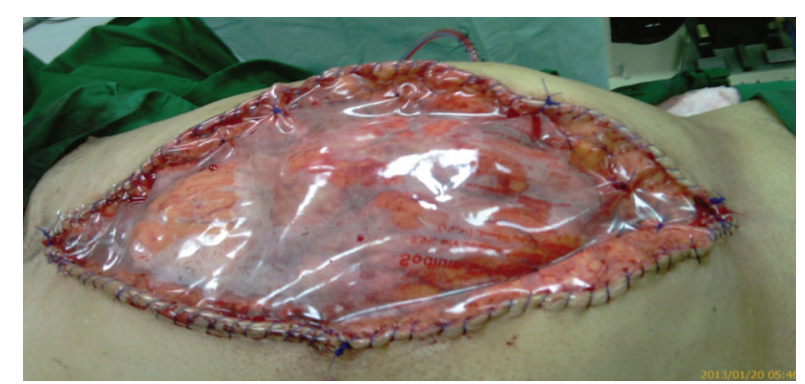

Figure (1): Operative photograph of temporary abdominal closure.

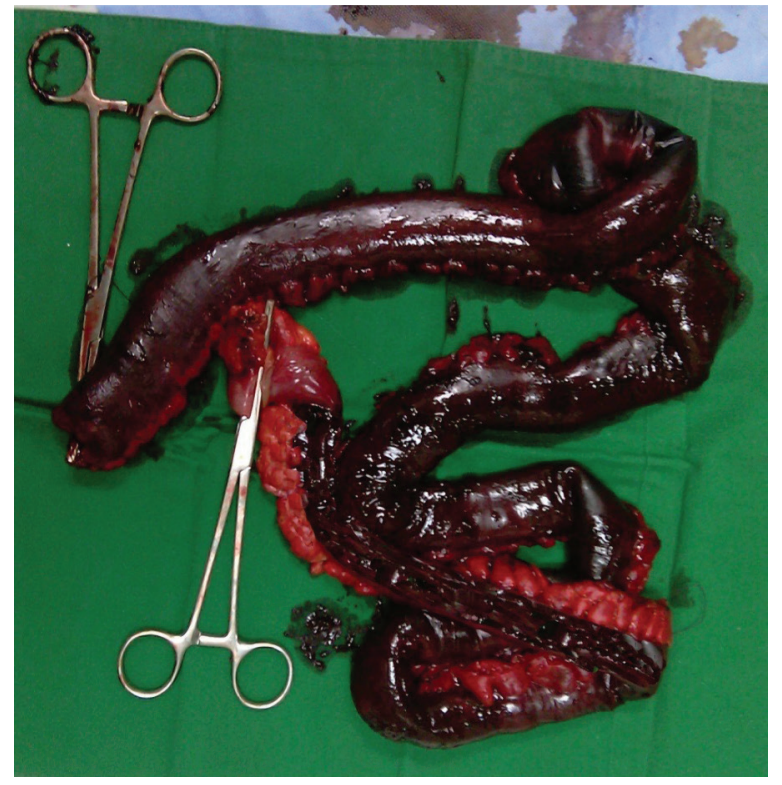

Figure (2): Operative photograph showing resected gangrenous jujenal loops after supersior mesenteric vein thrombosis following blunt abdominal trauma.

an important refuge to reduce morbidity and mortality in trauma resuscitation as it gives the patient a chance to survive in an otherwise hopeless situation. It is difficult to learn when to stop and can be learned only from experience. The results obtained from our study are consistent with other studies published to-date i.e. Reducing mortality and morbidity in addition to an improved outcome. The management of this complex problem requires a multidisciplinary team approach with patient counseling and communication with the family.

\section{Reference:}

1- Burch IM, Ortiz VB, Richardson RJ, Martin RR, Mattox KL, Jordan GL Jr: Abbreviated laparotomy and planned reoperation for critically injured patients. Ann Surg 1992: 215: 476-483.

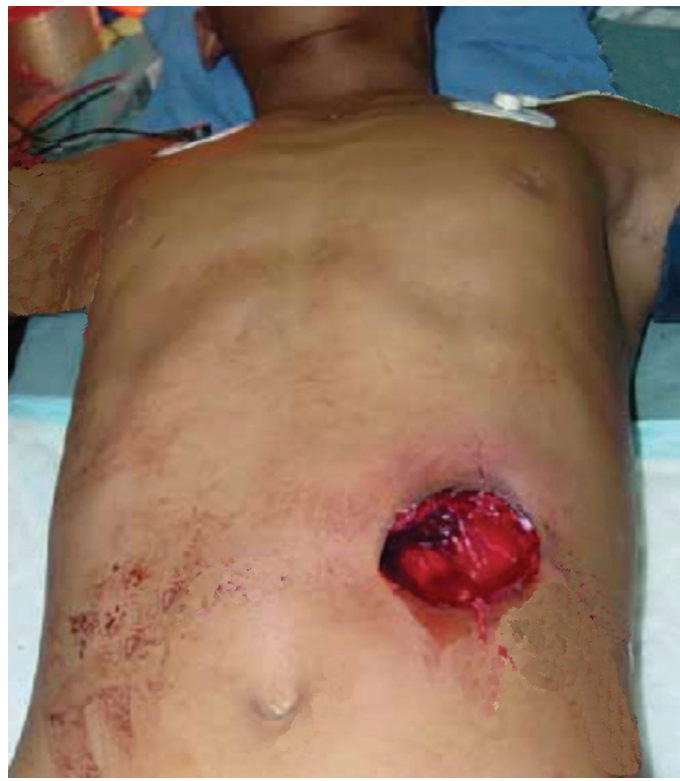

Figure (3): Abdominal automatic gunshot wound with resultant multiuple intraabdominal injuries nesscitated damage control approach laparotomy.

2- Rotondo M, Schwab C, McGonigal M, et al: Damage control: An approach for improved survival in exsanguinating penetrating abdominal injury. J Trauma 1993; 35(3): 375-382.

3- Moore EE, Burch JM, Francios RJ, et al: Staged physiologic restoration and damage control surgery. World J Surg 1998; 22(12): 1184-1190.

4- CW Schwab: Introduction: Damage control at the start of 21 st century. Editorial Int $J$ Care Injured 2004; 35: 639-641.

5- Johnson J, Gracias V, Schwab C, et al: Evolution in damage control in exsanguinating penetrating abdominal injury. J Trauma 2001; 51: 261-271.

6- Kouraklis G, Spirakos S, Glinavou A: Damage control surgery: An alternative approach for the management of critically injured patients. Surg Today 2002; 32: 195-202. 
Table 1: Detected abdominal organ injuries in 42 patients:

\begin{tabular}{|l|l|l|}
\hline \multicolumn{1}{|c|}{ Organ injury } & \multicolumn{1}{|c|}{$\begin{array}{c}\text { Percentage in the treated } \\
\text { patients }\end{array}$} & \multicolumn{1}{c|}{ Intervention } \\
\hline Liver & $45 \%(19$ patients $)$ & Perihepatic packing \\
\hline $\begin{array}{l}\text { Small intestine and } \\
\text { avulsed mesentery }\end{array}$ & $40 \%(17$ patients $)$ & Gut resection and temporary stapling \\
\hline Large intestine & $35.7 \%(15$ patients $)$ & $\begin{array}{l}\text { Colon resection and temporary } \\
\text { stapling }\end{array}$ \\
\hline Duodenum & $9.5 \%(4$ patients $)$ & Temporary external tube drainage \\
\hline Spleen & $16.7 \%(7$ patients $)$ & Splenectomy \\
\hline Kidney & $19 \%(8$ patients $)$ & Perinephric packing \\
\hline Inferior vena cava & $4.8 \%(2$ patients $)$ & Ligation \\
\hline Iliac vessels & $12 \%(5$ patients $)$ & Temporary shunting or ligation. \\
\hline Diaphragm & $9.5 \%(4$ patients $)$ & Packing \\
\hline Bladder and ureter & $12 \%(5$ patients $)$ & Packing and tie-off \\
\hline Stomach & $9.5 \%(4$ patients $)$ & Primary suturing \\
\hline Pancreas & $7 \%(3$ patients $)$ & Packing \\
\hline
\end{tabular}

7- Loveland JA, Boffard KD: Damage control in the abdomen and beyond. Br J Surg 2004; 91: 1095-1101.

8- American College of Surgeon's Committee on Trauma: Advanced Trauma Life Support Manual, 9th Edition, Chicago: ACS, 2012: 11-307.

9- Beekley AC: Damage control resuscitation: A sensible approach to the exsanguinating surgical patient. Crit Care Med 2008; 36: 267-274.

10- Mattox K: Introduction, background, and future projections of damage control surgery. Surg Clin North Am 1997; 77: 753-759.

11- Frank SM, Beattie C, Christopherson R, Norris EJ, Peraler BA, Williams GM, et al: Unintentional hypothermia is associated with post operative myocardial ischaemia. Anaesthesiology 1992; 78: 468-476.

12- Mitra B, Cameron PA, Mori A, Fitzgerald M: Acute coagulopathy and early deaths post major trauma. Injury 2012; 43(1): 22-25.

13- Waibel BH, Schlitzkus LL, Newell MA, et al: Impact of hypothermia (below 36 degrees $\mathrm{C}$ ) in the rural trauma patient. J Am Coll Surg 2009; 209: 580-588.

14- Rotondo MF, Zonies DH: Damage control sequence and underlying logic. Surg $\mathrm{Cl}$ North Am 1997; 77: 761-77.

15- Abramson D, Scalea TM, Hitchcock R, Trooskin SZ, Henry SM, Greenspan J: Lactic clearance and survival following injury. $J$ Trauma 1993; 35: 584-589.

16- Brohi K: Damage control surgery. Trauma Org 2000; 5: 6.

17- Asensio JA, Petrone P, Roldan G, Kuncir E, Ramicone E, Chan Linda: Has evolution in awareness of guidelines for institution of damage control improved outcome in the management of posttraumatic open abdomen? Arch Surg 2004; 139: 209-214.

18- Wyrzykowski AD, Feliciano DV: Trauma damage control. In: Feliciano DV, Mattox KL, Moore EE, editors. Trauma. $6^{\text {th }}$ ed. New York Chicago San Francisco: McGraw-Hill Companies; 2008; 851-870.

19- Gubler KD, Gentilello LM, Hassantash SA, Maier RV: The impact of hypothermia on dilutional coagulopathy. $J$ trauma 1994; 36: 847-855.

20- Gillespie DL, Cox ED, Kragh JF JR, Mehta SG, Salinas J, Holcomb JB: Damage control resuscitation for vascular surgery in a combat support hospital. J Trauma 2008; 63(1): 1-9. 